\title{
Tubular paramyxovirus-like structures in synovial vascular endothelium
}

\author{
H. RALPH SCHUMACHER, JR. \\ Arthritis Section, Department of Medicine, University of Pennsylvania, Philadelphia, Pa.
}

Chou (1967) first described aggregates of possibly viral tubular structures in muscle in polymyositis. Other workers have since found similar tubules in another 'collagen disease', systemic lupus erythematosus (Fresco, 1968; Györkey, Min, Sincovics, and Györkey, 1968; Hashimoto, 1969; Norton, 1969; Hurd, Eigenbrodt, Ziff, and Strunk 1969; Kawano, Miller, and Kimmelstiel, 1969). Most reports have emphasized these inclusions in kidney glomerular endothelium. Such structures have also been demonstrated in muscle, skin, and kidney peritubular cells but not in the synovium which is so commonly involved in the 'collagen diseases'. This communication reports identical tubular inclusions in synovial biopsies from patients with systemic lupus and transient undiagnosed synovitis.

\section{Methods}

As part of a study of normal and pathological synovial blood vessels, post mortem specimens from rabbits and monkeys and needle synovial biopsies from patients have been promptly placed in a formaldehyde-glutaraldehyde fixative diluted $1: 1$ with $0 \cdot 1 \mathrm{M}$ cacodylate buffer at $\mathrm{pH} 7 \cdot 4$ (Karnovsky, 1965), cut into $1 \times 1 \mathrm{~mm}$. pieces, fixed for
$4 \mathrm{hrs}$ at room temperature, washed in cacodylate buffer, post-fixed in cold Palade's osmium-veronal for $2 \mathrm{hrs}$, dehydrated with alcohol, and embedded in Epon 812. Ultrathin sections were stained with uranyl acetate and lead citrate and examined on an RCA EMU $3 \mathrm{H}$ electron microscope.

\section{Results}

No tubular inclusions were seen in many normal animal synovia despite specific attention directed at the microvasculature (Schumacher, 1969). Endothelial inclusions were found in only two diseased synovial membranes of the many examined.

(1) A 16-year-old girl had systemic lupus erythematosus. She had arthralgias without objective joint changes and no effusions. Synovial biopsy of the knee, however, showed focal synovial cell lining proliferation, vascular congestion with large endothelial cells and multilamination of some basement membranes, perivascular mononuclear cell infiltration, and perivascular and superficial fibrinlike material. The tubular aggregate seen by electron microscopy (Fig. 1) was in a venule that appeared otherwise normal. Diameter of the paramyxovirus-like tubules was approximately $220 \AA$.

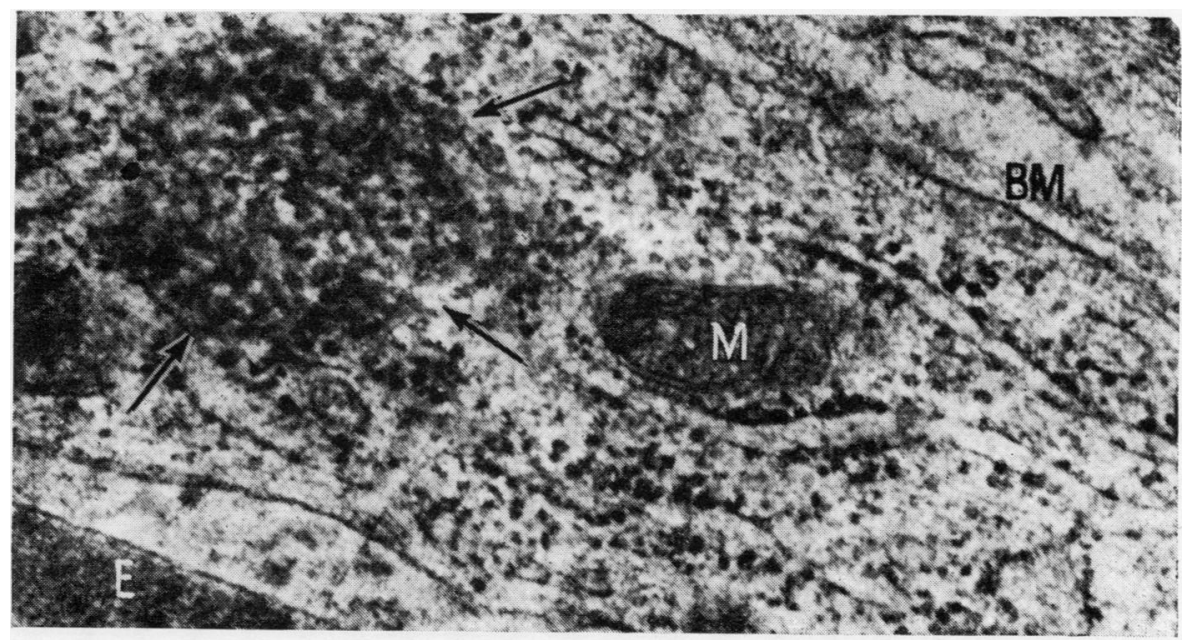

FIG. 1 Tubular aggregate (arrows) in venular endothelium of a 16-yeqr-old girl with systemic lupus erythematosus. $M$ mitochondrion.

$E$ erythrocyte in lumen.

$B M$ basement membrane. No membrane surrounds the tubules which lie near the rough endoplasmic reticulum. $\times 70,000$. 
(2) A 13-year-old boy had an undiagnosed, transient, inflammatory synovitis of the left knee of 1 week's duration. This followed 1 week after an upper respiratory infection and was associated with a temperature of $99 \cdot 8^{\circ} \mathrm{F}$. orally and a Wintrobe erythrocyte sedimentation rate of $49 \mathrm{~mm}$. 1st hour. Normal studies included antinuclear factor, L.E.-cell preparation, serum complement, antistreptolysin- 0 titre, latex-fixation test, and cultures for bacteria. Synovial cells were maintained in tissue culture for 6 months by Dr. Milan Fiala without evidence of viral activity. Like Case 1 he had received only occasional aspirin. The synovial fluid contained $14,000 \mathrm{WBC} / \mathrm{mm}^{3}$. with 66 per cent. lymphocytes. Synovial biopsy showed lining cell proliferation with phagocytic cells containing many vacuoles filled with finely granular material; focal perivascular mononuclear cell infiltration; and some vessels occluded with fibrin, platelets, and clumps of mononuclear cells. Vascular basement membranes were multilaminated. The tubular aggregates were formed in one of several enlarged endothelial cells that greatly narrowed the vascular lumen (Fig. 2). The basement membrane of this vessel was in disarray. There was no evident cytotoxic change.

Inclusions in both patients were in the cytoplasm adjacent to elements of the rough endoplasmic reticulum and had no other surrounding membranes. No continuity of tubules with cell membranes was found.

\section{Discussion}

Tubular inclusions similar to those described in other tissues in the 'collagen diseases' also occur in the synovium, although they are apparently less common than in the kidney. One patient with these inclusions had systemic lupus which has so far been the disease most frequently associated with these structures. The cause of the transient arthritis in the boy reported here is not known. Similar inclusions have been reported in scleroderma, discoid lupus erythematosus, Goodpasture's syndrome, and idiopathic thrombocytopenic purpura (Norton, 1969), but not yet in normal tissue. Ultra-structural study of the arthritis occurring in virus infection such as rubella or after rubella vaccination has not been reported. Little attention has as yet been directed to specifically searching for these structures in non-rheumatic diseases.

As discussed in other descriptions of these structures, the tubule diameter seems slightly greater than generally accepted sizes of known paramyxoviruses (Norton, 1969). Resolutions in our and other electron micrographs have as yet shown no helical structures. The absence of any consistent cell damage near the inclusions offers no evidence for any direct cytotoxic effect from these possible viruses, but an infecting agent still could provide an antigen leading to immunologic reactions. Viruses could also conceivably be secondary invaders allowed by altered resistance in the collagen diseases although our one patient had total clinical disease of only one week's duration. Virus cultures have so far been uniformly negative (Norton, 1969). It also remains possible that these inclusions are microtubules or other cell structures altered in a presently unrecog-

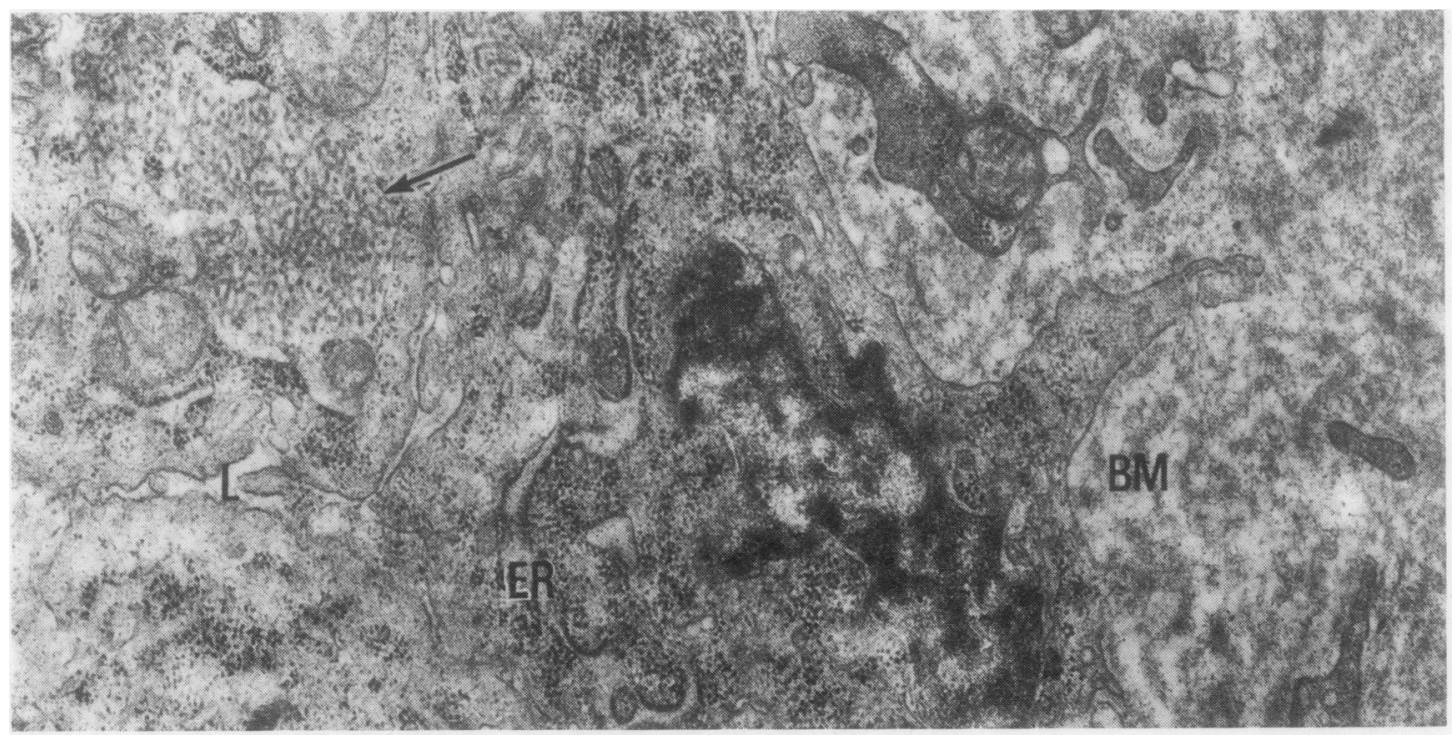

FIG. 2 Collection of tubular structures (arrow) surrounded by elements of rough endoplasmic reticulum in venular endothelium of a 13-year-old boy with transient undiagnosed synovitis.

$L$ vascular lumen.

$E R$ endoplasmic reticulum of adjacent endothelial cell.

BM thickened, disorganized membrane layer. $\times 26,000$. 
nized fashion. Hurd and others (1969) speculated that they might be cellular material released into the circulation and then phagocytosed by endothelial cells. Although endothelial cells can definitely phagocytose circulating particles (Schumacher, 1969), the tubular structures in the synovium have shown no surrounding phagosome membrane to support such a possible origin. In fact, the inclusions rather appear to be related to the rough endoplasmic reticulum. Known paramyxoviruses often lie in nucleii or free in the cytoplasm in parallel arrays rather than in the intermeshed networks typical of these endothelial inclusions. However, the cytoplasmic and nuclear tubules in the brains in subacute sclerosing panencephalitis from which measles virus has recently been isolated are somewhat interwoven (Chen, Watanabe, Zeman, and Mealey, 1969; Payne, Baublis, and Itabashi, 1969). Collections of tubules of approximately $240 \AA$ diameter but with a crystalline arrangement have have been reported in association with the endoplasmic reticulum in tissue cultures from various sources (Chandra, 1968). Bundles of $420 \AA$ tubules have been described in lymphocytes from one) patient with rheumatoid arthritis (Hovig, Jeremic, and Stavem, 1968).

\section{Summary}

Tubular aggregates, similar to those reported in other tissues in the 'collagen diseases', have been described lying adjacent to the endoplasmic reticulum in synovial vascular endothelium in patients with systemic lupus erythematosus and transient undiagnosed arthritis.

These recently identified vascular endothelial inclusions remain unexplained. Their possible viral nature is adding impetus to the continued search for an infectious agent as a factor in the pathogenesis of the systemic rheumatic diseases.

\section{References}

ChANDRA, S. (1968) Lab. Invest., 18, 422 (Undulating tubules associated with endoplasmic reticulum in pathologic tissues).

Chen, T. T., Watanabe, I., Zeman, W., and Mealey, J. (1969) Science, 163, 1193 (Subacute sclerosing panencephalitis: propagation of measles virus from brain biopsy in tissue culture).

CHOU, S. M. (1967) Ibid., 158, 1453 (Myxovirus-like structures in a case of human chronic polymyositis).

Fresco, R. (1968) Fed. Proc., 27, 246, Abstr. no. 170 (Tubular (myxovirus-like) structures in glomerular deposits from a case of lupus nephritis).

Györkey, F., Min, K. W., Sincovics, J. G., AND Györkey, P. (1968) New Engl. J. Med., 280, 333 (Systemic lupus erythematosus and myxovirus).

HASHIMOTO, K. (1969) "Paramyxovirus-like structures in lupus and dermatomyositis", in "Proc. 27th Annual Meeting of the Electron Microscopy Society of America", ed. C. J. Arcenaux, p. 222. Claitor's Publishing Division, Baton Rouge, Louisiana.

Hovig, T., Jeremic, R. T., And StaVem, P. (1968) Scand. J. Haemat., 5, 81 (A new type of inclusion bodies in lymphocytes).

Hurd, E. R., EIgenbrodt, E., Ziff, M. AND Strunk, S. W. (1969) Arthr. and Rheum., 12, 541 (Cytoplasmic tubular structures in kidney biopsies in systemic lupus erythematosus).

Karnovsky, M. J. (1965) J. Cell Biol., 27, 137A, Abstr. no. 270 (A formaldehyde-glutaraldehyde fixative of high osmolality for use in electron microscopy).

Kawano, K., Miller, L., And Kimmelstiel, P. (1969) New Engl. J. Med., 281, 1228 (Virus-like structures in lupus erythematosus).

Norton, W. L. (1969) J. Lab. clin. Med., 74, 369 (Endothelial inclusions in active lesions of systemic lupus erythematosus).

Payne, F. E., Baublis, J. V., and Itabashi, H. H. (1969) New Engl. J. Med., 281, 585 (Isolation of measles virus from cell cultures of brain from a patient with subacute sclerosing panencephalitis).

SCHUMACHer, H. R. (1969) Arthr. and Rheum., 12, 387 (The microvasculature of the synovial membrane of the monkey: Ultrastructural studies). 